\title{
Two-dimensional X-ray diffraction characterization of (Zn,Cd,Mg)Se wurtzite layers grown on $\mathrm{Bi}_{2} \mathrm{Se}_{3}$
}

\section{L.C. Hernandez-Mainet ${ }^{1}$, Z.Chen ${ }^{2,3}$, T. A. Garcia, ${ }^{1,3}$ A. B. Bykov², L. Krusin-Elbaum²,3 and M.C. Tamargo ${ }^{1,3, *}$}

\author{
${ }^{1}$ Chemistry Department, City College of New York, New York, NY 10031 \\ ${ }^{2}$ Physics Department, City College of New York, New York, NY 10031 \\ ${ }^{3}$ The Graduate Center CUNY, New York, NY 10016 \\ *Corresponding author: mtamargo@ccny.cuny.edu
}

\section{Abstract}

The wurtzite structure of $\mathrm{ZnSe}, \mathrm{Zn}_{0.49} \mathrm{Cd}_{0.51} \mathrm{Se}$ and $\mathrm{Zn}_{0.23} \mathrm{Cd}_{0.25} \mathrm{Mg}_{0.52} \mathrm{Se}$ layers grown on $\mathrm{Bi}_{2} \mathrm{Se}_{3} /$ sapphire (0001) by Molecular Beam Epitaxy (MBE) is reported. Structure characterization is studied by two-dimensional X-ray diffraction. Pole figures are calculated for cubic and hexagonal planes of the $(\mathrm{Zn}, \mathrm{Cd}, \mathrm{Mg}) \mathrm{Se}$ family and compared to their expected values. The targeted wurtzite plane was (11-22), while the cubic ones were the (220) and (311). The results show that, under our MBE growth conditions, $\mathrm{ZnSe}, \mathrm{Zn}_{0.49} \mathrm{Cd}_{0.51} \mathrm{Se}$ and $\mathrm{Zn}_{0.23} \mathrm{Cd}_{0.25} \mathrm{Mg}_{0.52} \mathrm{Se}$ layers prefer to form the hexagonal (wurtzite) phase rather than the cubic one when grown on $\mathrm{Bi}_{2} \mathrm{Se}_{3} /$ sapphire in (0001) direction. These results have implications for the next generation devices combining semiconductors and topological insulator materials.

Keywords - Topological Insulator, Semiconducting II-VI materials, two dimensional XRD, Molecular beam epitaxy; 


\section{Introduction}

Topological insulators are of interest for the next generation solid state devices ${ }^{1}$. Among topological insulators, bismuth selenide $\left(\mathrm{Bi}_{2} \mathrm{Se}_{3}\right)$ is receiving attention for its unique topological features and promise in novel applications ${ }^{2}$. Exotic behaviors arising from topological orders are predicted to be enhanced in structures comprising alternating layers of $\mathrm{Bi}_{2} \mathrm{Se}_{3}$ and band insulators. Thus, combining $\mathrm{Bi}_{2} \mathrm{Se}_{3}$ with conventional semiconductors in heterostructures will provide an exciting new dimension in materials design ${ }^{3}$. Recently new $(\mathrm{Zn}, \mathrm{Cd}, \mathrm{Mg}) \mathrm{Se}$-based II-VI semiconductor/ $\mathrm{Bi}_{2} \mathrm{Se}_{3}$ heterostructures grown by molecular beam epitaxy (MBE) have been reported in our group ${ }^{4}$. The ( $\left.\mathrm{Zn}, \mathrm{Cd}, \mathrm{Mg}\right) \mathrm{Se}$ family of II-VI materials was chosen to combine with $\mathrm{Bi}_{2} \mathrm{Se}_{3}$ with the expectation that chemical compatibility of selenides will facilitate growth. In addition, they have comparable optimal MBE growth conditions, a small lattice mismatch and tunable bandgaps, making them ideal candidates for heterostructures grown by this technique. Despite the outstanding results reported, further analysis of the epitaxial relationships between the II-VI semiconductors and $\mathrm{Bi}_{2} \mathrm{Se}_{3}$ is needed to obtain a better understanding and optimization of their physical properties.

In conventional epitaxial growth processes, the growing layer typically exhibits the same crystal structure and the same orientation as the layer that lays beneath ${ }^{5}$. However, the van der Waals bonding nature of the $\mathrm{Bi}_{2} \mathrm{Se}_{3}$ material is expected to exert less influence on the overlayer structure since the van der Waals materials do not form interlayer covalent bonds ${ }^{6}$. The $\mathrm{Bi}_{2} \mathrm{Se}_{3}$ structure is described in terms of a layered hexagonal primitive cell with three, five-atom thick layers, often referred to as a quintuple layer ${ }^{7}$. On the other hand, a zinc blend three-dimensional structure is typically preferred by the $(\mathrm{Zn}, \mathrm{Cd}, \mathrm{Mg}) \mathrm{Se}$ family rather than the hexagonal wurtzite structure. It has been suggested that under some MBE growth conditions, the hexagonal phase may be favored ${ }^{8,9,10}$ over the zinc blend, even when the latter may be thermodynamically favored. In this paper the preferred crystal structure (zinc blend or wurtzite) of II-VI semiconductors grown by $\mathrm{MBE}$ on $\mathrm{Bi}_{2} \mathrm{Se}_{3}$ is studied through the plotting of pole figures using a two-dimensional X-ray detector.

The data for pole figure calculations is often collected through a number of rotation scans $(\phi)$ at different tilted angles $(\psi)$, and at $2 \theta$ fixed to the Bragg angle of the desired crystallographic plane using a point detector, a lengthy and time consuming process. Two-dimensional X-ray diffraction $\left(\mathrm{XRD}^{2}\right)$ provides advantages when plotting pole figures over X-ray diffraction. The $\mathrm{XRD}^{2}$ refers to X-ray diffraction application using a two-dimensional (2D) detector. Two-dimensional detector collects diffracted X-rays in a large angular range in both $2 \theta$ and $\Psi$ directions. The number of sample tilts required for complete pole 
figure calculation is much less than with point detector. Moreover, diffraction from a number of crystallographic planes is collected simultaneously for a fixed $2 \mathrm{D}$ detector position due to a wide $2 \theta$ range of the detector. Therefore, $\mathrm{XRD}^{2}$ measurements reduce the number of scan steps used to achieve a full pole figure, saving a considerable data collection time. In addition to the 2D detector technology, the plotting of pole figures involves processing of images and interpretation. The basics and advantages of X-ray diffraction measurements using a 2D area detector have been reported elsewhere ${ }^{11}$.

In this paper, pole figures are calculated by $\mathrm{XRD}^{2}$ techniques for different cubic and hexagonal planes for $\mathrm{ZnSe}$, $\mathrm{Zn}_{0.49} \mathrm{Cd}_{0.51} \mathrm{Se}$ and $\mathrm{Zn}_{0.23} \mathrm{Cd}_{0.25} \mathrm{Mg}_{0.52} \mathrm{Se}$ layers grown on $\mathrm{Bi}_{2} \mathrm{Se}_{3}$ layers grown on (0001) sapphire substrates. The selected hexagonal plane is (11-22), while the cubic ones are the (220) and (311). In addition, the same set of planes was examined on a sample of $\mathrm{Bi}_{2} \mathrm{Se}_{3}$ grown on sapphire and one of the pure bulk (0001) sapphire (the substrate). These additional measurements allow us to identify the pole figure signals originating from those other two materials. We find that under our growth conditions, the II-VI semiconductor layers exhibit a predominantly wurtzite structure, which may be a desirable feature for some applications.

\section{Experimental details}

Three samples (T37, T39 and T40) were investigated by the plotting of pole figures. In each case, single crystalline layers of $\mathrm{ZnSe}$ (T37), $\mathrm{Zn}_{0.49} \mathrm{Cd}_{0.51} \mathrm{Se}$ (T39) or $\mathrm{Zn}_{0.23} \mathrm{Cd}_{0.25} \mathrm{Mg}_{0.52} \mathrm{Se}$ (T40) semiconductors were grown on a $6 \mathrm{~nm}$ layer of $\mathrm{Bi}_{2} \mathrm{Se}_{3}$ previously deposited on (0001) sapphire substrates by MBE. In addition a $6 \mathrm{~nm}$ single layer of $\mathrm{Bi}_{2} \mathrm{Se}_{3}$ was grown on a sapphire substrate (T21), with no II-VI overlayers. The experimental details of the MBE growth have been reported elsewhere ${ }^{4}$. The compositions of T39 and T40 were chosen to minimize the in-plane lattice mismatch between the II-VI overlayer and the $\mathrm{Bi}_{2} \mathrm{Se}_{3}$. The XRD ${ }^{2}$ data were collected on a Bruker Discover 8 X ray diffraction system with VANTEC-500 area detector. The system utilizes a parallel incident X-ray beam collimated by Gobbel mirror and $0.5 \mathrm{~mm}$ pinhole collimator. The detector was kept $200 \mathrm{~mm}$ away from the sample. Data collection simultaneously takes place in a wide $2 \theta$ range of about $30^{\circ}$

In a pole figure measurement, the diffraction angle $(2 \theta)$ is fixed for a targeted plane $\left(h_{2} k_{2} l_{2}\right)$. Then, the sample is tilted at an angle $\psi$ from the sample surface normal direction $\left(h_{l} k_{l} l_{1}\right)$. For cubic and hexagonal systems, $\psi$ may be found from the following equations 1 and 2 , respectively ${ }^{12}$ : 


$$
\begin{gathered}
\cos \psi=\frac{h_{1} h_{2}+k_{1} k_{2}+l_{1} l_{2}}{\sqrt{\left({h_{1}}^{2}+{k_{1}}^{2}+{l_{1}}^{2}\right)\left({h_{2}}^{2}+{k_{2}}^{2}+l_{2}{ }^{2}\right)}} \\
\cos \psi=\frac{h_{1} h_{2}+k_{1} k_{2}+\frac{1}{2}\left(h_{1} k_{2}+h_{2} k_{1}\right)+\frac{3 a^{2}}{4 c^{2}} l_{1} l_{2}}{\sqrt{\left(h_{1}{ }^{2}+{k_{1}}^{2}+h_{1} k_{1}+\frac{3 a^{2}}{4 c^{2}} l_{1}{ }^{2}\right)\left({h_{2}}^{2}+{k_{2}}^{2}+h_{2} k_{2}+\frac{3 a^{2}}{4 c^{2}} l_{2}{ }^{2}\right)}},
\end{gathered}
$$

where $a$ and $c$ are the lattice constant and the height, respectively, for the corresponding hexagonal structure. Once $2 \theta$ and $\psi$ are fixed, the sample is exposed to the X-ray beam while it rotates an $\phi$ angle around to the surface normal. Measurement results are saved as an image (frame).

To cover all plane reflections, the sample is rotated from $0^{\circ}$ to $360^{\circ}$ with a scan step of $10^{\circ}$. This implies that 36 frames will be collected during the full $\psi$ rotation. The 36 frames provide a large amount of information for calculating the pole figures. In order to analyze the data, MULTEX by Bruker software allows loading a set of frames for the calculation of pole figures for any selected $2 \theta$ angle $^{13}$. Furthermore, PILOT by Bruker software can integrate frame intensities into a one dimensional profile which provides a plot similar to conventional "Intensity vs. $2 \theta$ " diffraction pattern. To identify the peaks, Powder Diffraction File (PDF) card information was used for $\mathrm{ZnSe}$ and $\mathrm{Zn}_{0.5} \mathrm{Cd}_{0.5} \mathrm{Se}$. The quaternary material, $\mathrm{Zn}_{0.23} \mathrm{Cd}_{0.25} \mathrm{Mg}_{0.52} \mathrm{Se}$ studied here has a similar lattice constant to $\mathrm{Zn}_{0.5} \mathrm{Cd}_{0.5} \mathrm{Se}$, so the PDF card values for $\mathrm{Zn}_{0.5} \mathrm{Cd}_{0.5} \mathrm{Se}$ were used also for $\mathrm{Zn}_{0.23} \mathrm{Cd}_{0.25} \mathrm{Mg}_{0.52} \mathrm{Se}$.

An example of the pole figure calculation procedure described above is illustrated in Fig. 1 for a bulk (0001) sapphire substrate. Fig 1(a) displays a typical frame with the raw data for the $\mathrm{XRD}^{2}$ measurement. The bright spot within the highlighted sector is a diffraction point from planes of sapphire single crystal. A bright spot is representative of single crystal diffraction in $\mathrm{XRD}^{2}$ measurement. To convert the 2D data into a XRD pattern, the sector is integrated and transformed into a $(\theta-2 \theta)$ pattern, as shown Fig 1(b). The graph displays a sharp peak at $53.8^{\circ}$ which belongs to sapphire (20-24) plane ${ }^{14}$. Once the peak is selected, the program runs the same $2 \theta$ range over all 36 frames. The calculated pole figure is shown in Fig 1 (c), displaying three poles at about $\psi=60^{\circ}$. These tree poles are explained by the three-fold rotational symmetry of sapphire (R- 
$\underline{3} \mathrm{C}$ space group), while the $\psi$ position corresponds to an angle of $57.6^{\circ}$ formed between the (20-24) plane and the (0001) surface normal plane (see Eq.2). Thus, three important parameters are needed to identify a targeted plane: the $2 \theta$ diffraction peak, the $\psi$ position, and the number of reflections or "poles".

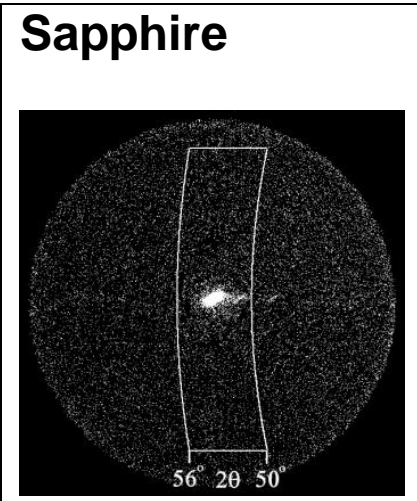

(a)

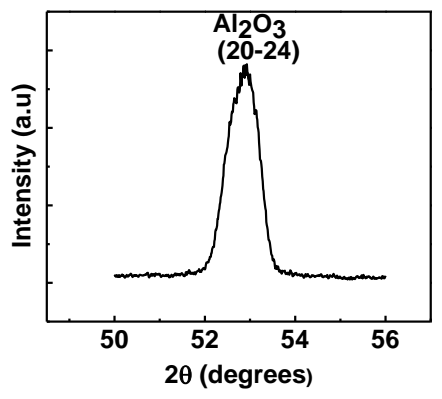

(b)

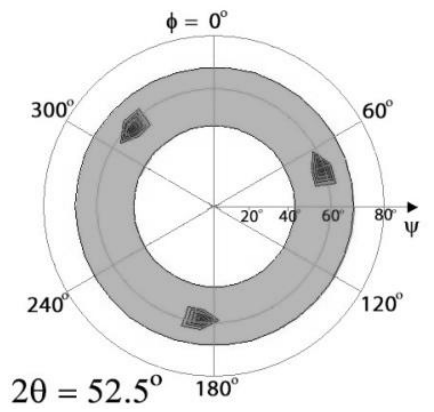

(c)

Fig.1. Measurement scheme for pole figures using the $\mathrm{XRD}^{2}$ technique for a bulk a (0001) sapphire substrate. (a) XRD ${ }^{2}$ data for one frame. The $2 \theta$ sector is highlighted. (b) The diffraction pattern as a result of integration in the highlighted sector. (c) Pole figure for (20-24) sapphire calculated over the 36 frames.

\section{Results and discussion}

Pole figures were computed for the three samples T37, T39 and T40. Fig.2 (a) displays the frame (inset) and its integrated diffraction pattern of the (11-22) plane in ZnSe measured for T37. The integrated diffraction pattern of ZnSe displays a clear peak at $53.8^{\circ}$, which is assumed to belong to the $\mathrm{ZnSe}(11-22)$ plane ${ }^{15}$. The calculated pole figure over the same $2 \theta$ range in Fig. $2 \mathrm{~b}$ shows six clear poles at about $\psi=58.6^{\circ}$ which matches with the calculated value for the (11-22) plane in $\mathrm{ZnSe}$ structure. This plane clearly shows its hexagonal features: $2 \theta=53.7^{\circ}, \psi=58.6^{\circ}$ and 6 reflections, supporting the identification of the wurtzite phase. Two poles with weak intensity (dashed circles) can be also seen in Fig. $2 \mathrm{~b}$ at about $\psi=$ $70, \phi=50^{\circ}$ and $\psi=60, \phi=290^{\circ}$, which are assigned to traces of diffraction from the $\mathrm{Al}_{2} \mathrm{O}_{3}(20-24)$ and $\mathrm{Bi}_{2} \mathrm{Se}_{3}(20-25)$ planes $^{14,16}$, respectively. This assignment is based on the pole figure measurement results obtained for $\mathrm{Bi}_{2} \mathrm{Se}_{3}$ on sapphire (sample T21) which is shown in Fig. 2c. In Fig. 2c, the outer poles presumably belong to $\mathrm{Bi}_{2} \mathrm{Se}_{3}(20-25)$ at $\psi=72.7^{\circ}$, while the inner poles correspond to $\mathrm{Al}_{2} \mathrm{O}_{3}$ (20-24) (as shown in Fig. 1c for pure sapphire). 


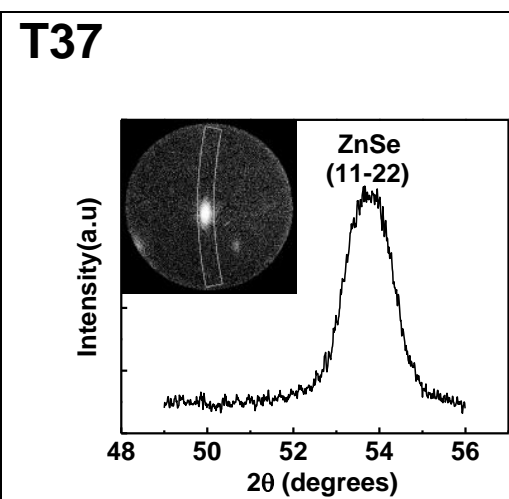

(a)

T21

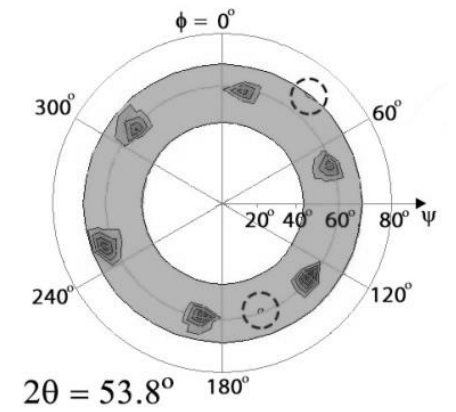

(b)

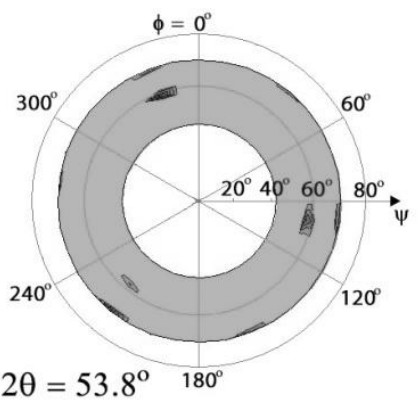

(c)

Fig 2 The diffraction peak profile after frame integration (a) and pole figure (b) of the (11-22) plane in ZnSe (T37). (c) Pole figures for $\mathrm{Bi}_{2} \mathrm{Se}_{3} / \mathrm{Al}_{2} \mathrm{O}_{3}$ (T21) using the same experimental conditions as for the (11-22) ZnSe plane.

Similar results are presented for $\mathrm{Zn}_{0.49} \mathrm{Cd}_{0.51} \mathrm{Se}$ (T39) and $\mathrm{Zn}_{0.23} \mathrm{Cd}_{0.25} \mathrm{Mg}_{0.52} \mathrm{Se}$ (T40) when the (11-22) plane was examined and they are shown in Fig. 3a and 3b, respectively. Both materials display a clear diffraction peak at about $2 \theta=51.8^{\circ}$ for $(11-$ 22) plane ${ }^{17}$. The insets show the frame raw data for each sample. The calculated pole figures over the $2 \theta$ range show six clear poles at about $\psi=58.6^{\circ}$ for $(11-22)$, also demonstrating the hexagonal phase of these layers. The $2 \theta$ value, $\psi$ position, and 6 reflections in both samples allow to identify the targeted (11-22) plane in the ( $\mathrm{Zn}, \mathrm{Cd}, \mathrm{Mg}) \mathrm{Se}$ family, supporting the conclusion that the wurtzite phase is present. In addition, the pole figure of Fig. $3 \mathrm{a}$ for the T39 sample displays an extra pole at about $\psi=60^{\circ}, \phi=220^{\circ}$ assigned to the sapphire (20-24) plane. In T40 (Fig. 3b), the $\mathrm{Bi}_{2} \mathrm{Se}_{3}(20-25)$ and $\mathrm{Al}_{2} \mathrm{O}_{3}(20-24)$ poles are more clearly seen at $\psi=72.7^{\circ}$ and $\psi=60^{\circ}$, respectively.

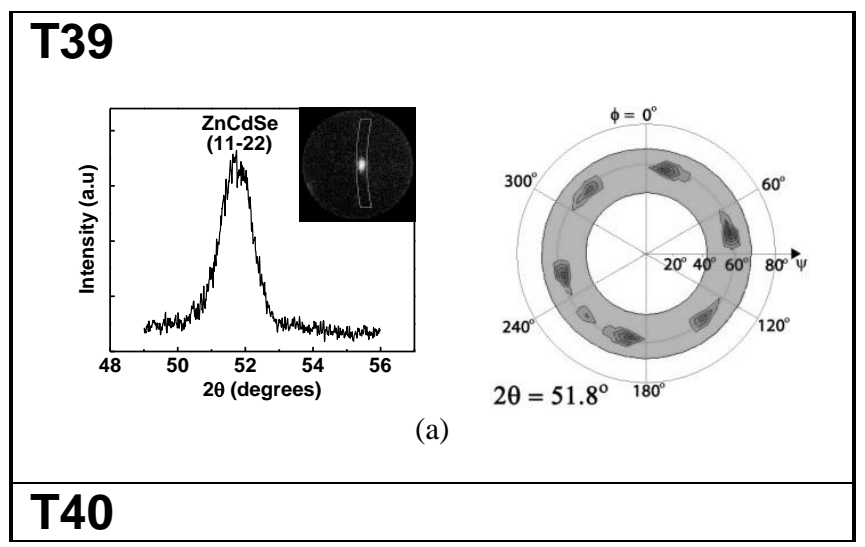




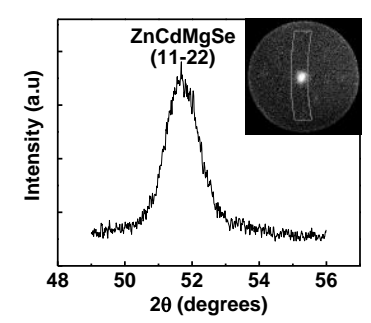

(b)

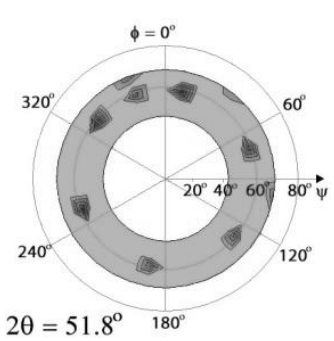

$2 \theta=51.8^{\circ} \quad 180$

Fig.3. The frame inset in its corresponding integrated diffraction pattern, and pole figures from (11-22) plane for (a) $\mathrm{Zn}_{0.49} \mathrm{Cd}_{0.51} \mathrm{Se}$ (T39), and (b) $\mathrm{Zn}_{0.23} \mathrm{Cd}_{0.25} \mathrm{Mg}_{0.52} \mathrm{Se}$ (T40).

Fig.4 shows the diffraction peak profile (after frame integration) and the pole figures corresponding to the cubic (220) plane ${ }^{18,19}$ for $\mathrm{ZnSe}$ (a), $\mathrm{Zn}_{0.49} \mathrm{Cd}_{0.51} \mathrm{Se}$ (b), and $\mathrm{Zn}_{0.23} \mathrm{Cd}_{0.25} \mathrm{Mg}_{0.52} \mathrm{Se}$ (c). From the integrated diffraction pattern of $\mathrm{ZnSe}(\mathrm{T} 37)$ two peaks at about $40.1^{\circ}$ and $42.9^{\circ}$ are seen, which are identified as the $\mathrm{Bi}_{2} \mathrm{Se}_{3}$ (10-1 10), and (10-1 11) planes. Also, one broad peak is observed between $45^{\circ}$ and $50^{\circ}$. Over this wide range an ambiguity arises between (220) and (10-13) of $\mathrm{ZnSe}$ due to their proximal $2 \theta$ values of $45.2^{\circ}$ and $49.5^{\circ}$, respectively. The pole figure made over this $2 \theta$ range shows six poles with about equal intensity, which suggests we are in the presence of a hexagonal system rather than a cubic system. Thus we conclude that we are seeing the pole figure for the hexagonal (10-13) plane of $\mathrm{ZnSe}$. If a twinned cubic phase were present we would expect to see two sets of three alternating poles of different intensities.

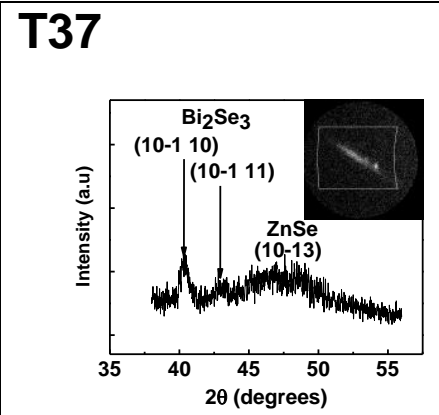

(a)

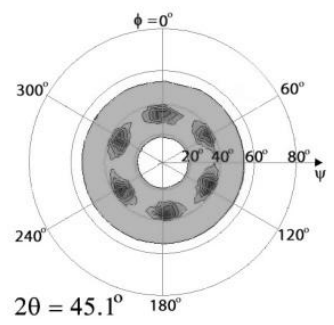

$2 \theta=45.1^{\circ} \quad 180^{\circ}$

\section{T39}
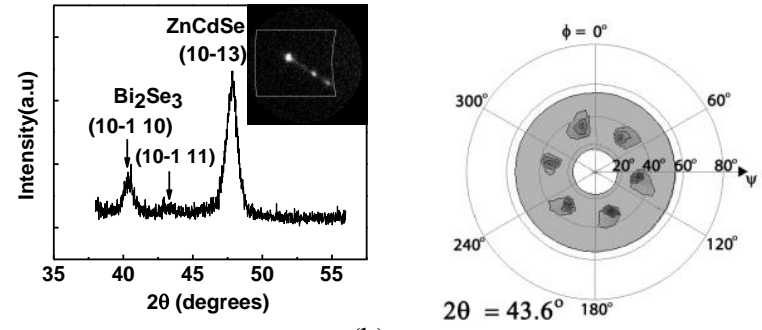

(b) 


\section{T40}
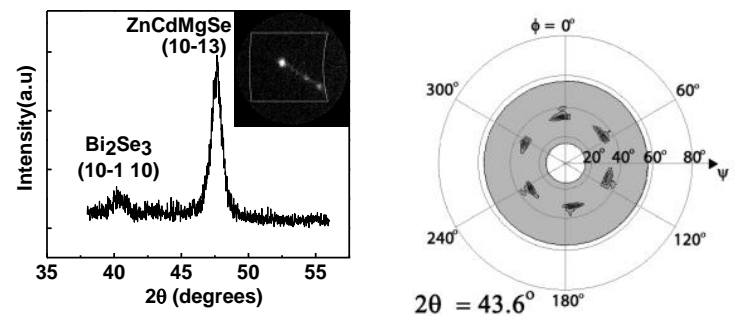

(c)

Fig.4. Integrated diffraction pattern with its frame (inset) and pole figures from (220) cubic plane for (a) $\mathrm{ZnSe}$ (T37), (b) $\mathrm{Zn}_{0.49} \mathrm{Cd}_{0.51} \mathrm{Se}$ (T39), and (c) $\mathrm{Zn}_{0.23} \mathrm{Cd}_{0.25} \mathrm{Mg}_{0.52} \mathrm{Se}$ (T40).

The integrated diffraction pattern from $\mathrm{Zn}_{0.49} \mathrm{Cd}_{0.51} \mathrm{Se}(\mathrm{T} 39)$ and $\mathrm{Zn}_{0.23} \mathrm{Cd}_{0.25} \mathrm{Mg}_{0.52} \mathrm{Se}$ (T40), shown in $4 \mathrm{~b}$ and $4 \mathrm{c}$, respectively, display a sharp peak at $47.7^{\circ}$ which originates from the (10-13) plane. Furthermore, a low intensity broad peak is shown, assigned to the previously observed plane of $\mathrm{Bi}_{2} \mathrm{Se}_{3}(10-110)$. There are no peaks for the (220) plane in either of the integrated patterns, expected at $2 \theta=43.5^{\circ}$, suggesting again the absence of the cubic phase The pole figure from T39 shows six broad poles due to the overlapping of $\mathrm{Zn}_{0.49} \mathrm{Cd}_{0.51} \mathrm{Se}(10-13)$ and $\mathrm{Bi}_{2} \mathrm{Se}_{3}(10-110)$ at $\psi=32.2^{\circ}$ and $38.7^{\circ}$, respectively. This overlapping is more clearly evident in T40 where the six poles of the (10-13) plane are surrounded by three low intensity poles at slightly higher position, which belong to $\mathrm{Bi}_{2} \mathrm{Se}_{3}(10-110)$ at $\psi=38.7^{\circ}$. The results show no evidence of the (220) plane: neither the $2 \theta$ peak nor the poles are seen. Thus, no evidence for the ( $\mathrm{Zn}, \mathrm{Cd}, \mathrm{Mg})$ Se cubic features were obtained, while the presence of the wurtzite structure is reinforced.

Finally, the (311) plane is examined in each sample and their integrated patterns and pole figures are displayed in Fig. 5 for (a) T37, (b) T39 and (c) T40. No peaks are displayed for the (311) plane in any of the $2 \theta$ diffraction patterns. In Fig 5c, for sample T40, a sharp peak at $2 \theta=47.7^{\circ}$ is seen which is consistent with the (10-13) wurtzite plane of $\mathrm{Zn}_{0.23} \mathrm{Cd}_{0.25} \mathrm{Mg}_{0.52} \mathrm{Se}_{\text {. }}$ The pole figures for all three samples display six poles with the same intensity just above $\psi=30^{\circ}$ that we index for $(10-13)$, according to previous results. Since neither the $2 \theta$ peak nor the poles are observed from the (311) plane, we conclude that there is no evidence for cubic features. 


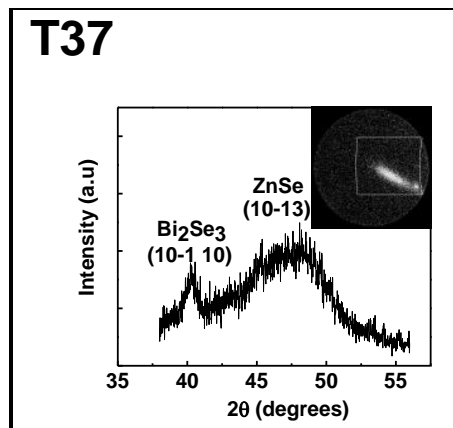

(a)

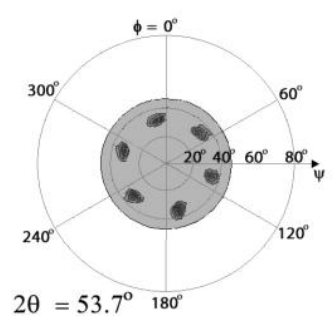

\section{T39}
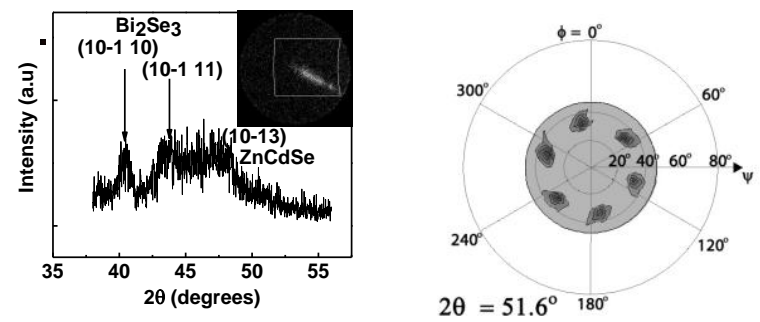

(b)

\section{T40}
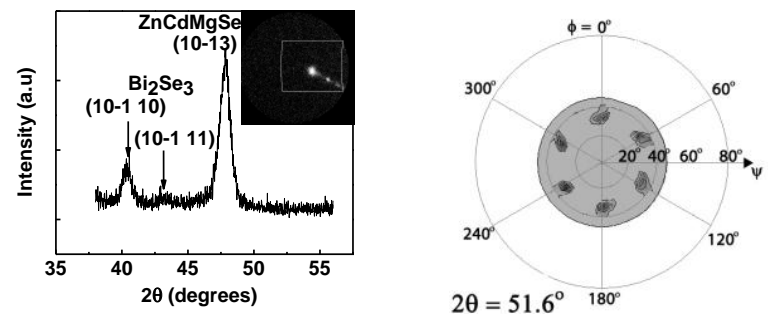

(c)

Fig.5. Frame (inset), integrated diffraction pattern and pole figures from the (311) cubic plane for (a) $\mathrm{ZnSe}$ (T37), (b) $\mathrm{Zn}_{0.49} \mathrm{Cd}_{0.51}$ Se (T39), and (c) $\mathrm{Zn}_{0.23} \mathrm{Cd}_{0.25} \mathrm{Mg}_{0.52} \mathrm{Se}$ (T40).

The results described above support the formation of a predominant metastable wurtzite phase in the three II-VI crystalline semiconductor layers grown on $\mathrm{Bi}_{2} \mathrm{Se}_{3}$ by MBE. These results suggest that the hexagonal symmetry of the $\mathrm{Bi}_{2} \mathrm{Se}_{3}$ surface allows the 3D II-VI crystal over layer to develop the wurtzite structure even when it is not the thermodynamically most stable surface. Wurtzite structures possess desirable properties that are different from their zinc-blend counterparts such as fewer glide planes and a polar band structure that may prove advantageous for some device applications. The possibility of intentionally stabilizing and optimizing the wurtzite phase of the II-VI overlayers by the appropriate choice of growth conditions ${ }^{10}$ is under investigation in our laboratory. 


\section{Conclusions}

The preferred crystal structure of $\mathrm{ZnSe}, \mathrm{Zn}_{0.49} \mathrm{Cd}_{0.51} \mathrm{Se}$, and $\mathrm{Zn}_{0.23} \mathrm{Cd}_{0.25} \mathrm{Mg}_{0.52} \mathrm{Se}$ layers grown by $\mathrm{MBE}$ on $\mathrm{Bi}_{2} \mathrm{Se}_{3}$ layers grown on sapphire were studied by two-dimensional XRD. To achieve this goal, pole figures were obtained for several cubic and hexagonal planes of the $(\mathrm{Zn}, \mathrm{Cd}, \mathrm{Mg}) \mathrm{Se}$ family. The results displayed the presence of a predominant wurtzite phase through the observation of the (11-22) and (10-13) planes, showing their $2 \theta$ value and six poles at the corresponding $\psi$ positions. On the other hand, there was no evidence of the cubic (220) and (311) planes: neither a peak at the appropriate $2 \theta$ values, nor the corresponding poles at $\psi$ positions were observed for those two planes. We conclude that under the MBE growth conditions employed, the II-VI layers grown on $\mathrm{Bi}_{2} \mathrm{Se}_{3}$-on-sapphire substrates exhibit a predominant wurtzite structure. The potential of stabilizing the wurtzite crystal structure in the $(\mathrm{Zn}, \mathrm{Cd}, \mathrm{Mg}) \mathrm{Se}$ material system opens new fields for fundamental physics and devices design.

\section{Acknowledgments}

This work was supported by the NSF Grant Nos. DMR-1122594 (MIRT), HRD-0833180 ((CREST Center for the Exploitation of Nanostructures for Sensors and Energy Systems, CENSES), and by DMR-1420634 (CCNY-Columbia MRSEC: Center for Precision Assembly of Superstratic and Superatomic Solids, PAS ${ }^{3}$ ). We also would like to acknowledge helpful discussions and insights of Prof. M. Kobayashi of Waseda University.

\section{References}

${ }^{1}$ L. He, X. Kou, and K. L. Wang. "Review of 3D topological insulator thin-film growth by molecular beam epitaxy and potential applications". Physics Status Solidi RRL 7, No. 1-2, 50-63 (2013).

${ }^{2}$ Z.-H. Pan, A. V. Fedorov, D. Gardner, Y. S. Lee, S. Chu and T. Valla "Measurement of an exceptionally weak electronphonon coupling on the surface of the topological insulator $\mathrm{Bi}_{2} \mathrm{Se}_{3}$ using angle-resolved photoemission spectroscopy", Physical Review Letters 108, 187001-1, (2012).

${ }^{3}$ R. Yoshimi, A. Tsukazaki, K. Kikutake, J. G. Checkelsky, K. S. Takahashi, M. Kawasaki1 and Y. Tokura, "Dirac electron states formed at the heterointerface between a topological insulator and a conventional semiconductor", Nature Materials 13, (2014).

${ }^{4}$ Z. Chen, T. A. Garcia, L. C. Hernandez-Mainet, L. Zhao, H. Deng, L. Krusin-Elbaum and M. C. Tamargo, "MBE growth and characterization of $\mathrm{Bi}_{2} \mathrm{Se}_{3} / \mathrm{II}-\mathrm{VI}$ semiconductor heterostructures", Applied Physics Letters 105, 242105, (2014). 
${ }^{5}$ M.A. Hermand and H.Sitter. "Molecular Beam Epitaxy. Fundamentals and Current Status", Springer Series in Materials Science, $2^{\text {nd }}$ edition, (1996).

${ }^{6} \mathrm{Y}$. Takagaki and B. Jenichen. "Epitaxial growth of $\mathrm{Bi}_{2} \mathrm{Se}_{3}$ layers on InP substrates by hot wall epitaxy". Semiconductor Science and Technology 27. 035015 (2012).

${ }^{7}$ B. Yan and S.-C. Zhang, “Topological materials”, Reports on Progress Physics 75, 1 (2012).

${ }^{8}$ H. D. Li, Z. Y. Wang, X. Guo, T. L. Wong, N. Wang, and M. H. Xie. "Growth of multilayers of Bi $\mathrm{Se}_{3} / \mathrm{ZnSe}$ : Heteroepitaxial interface formation and strain”, Applied Physics Letters 98, 043104 (2011);

${ }^{9}$ H. Umeya, K. Kitamura, A. Jia, M. Shimotomai, Y. Kato, M. Kobayashi, A. Yoshikawa and K. Takahashi, “MBE Growth and Characterization of Hexagonal ZnCdSe Layers on GaAs(111)-A and -B Substrates", . Physica Status Solidi (a) 192 (1), 195-200, (2002).

${ }^{10}$ S. Suzuki, Y. Kaifuchi, H. Kumada, Y. Ishitani and A. Yoshikawa, "MBE growth and characterization of hexagonal ZnCdMgSe layers and ZnCdSe/ZnCdMgSe QW structures on GaAs (111) substrates”, Physica Status Solidi (b) 24 (3), 475 , (2004).

${ }^{11}$ B. B. He. “Two-Dimensional X-Ray Diffraction”, John Wiley \& Sons, Inc, (2009).

${ }^{12}$ B. D. Cullity. "Elements of X-Ray Diffraction”, Addison-Wesley, Inc. (1956)

${ }^{13}$ K. Helming, M. Lyubchenko, B. He, and U. Preckwinkel. “A new method for texture measurements using a general area detector diffraction system", Powder Diffraction 18 (2). June (2003).

${ }^{14}$ JCPDS. Powder Diffraction File 5-0712.

${ }^{15}$ JCPDS.Powder Diffraction File 15-0105.

${ }^{16}$ JCPDS. Powder Diffraction File 1-077-7147.

${ }^{17}$ JCPDS. Powder Diffraction File 1-079-3173.

${ }^{18}$ JCPDS. Powder Diffraction File 1-071-5977.

${ }^{19}$ JCPDS. Powder Diffraction File 01-071-7293. 